\title{
Should topiramate use be added to the differential diagnosis of visual illusions?
}

\section{O uso do topiramato deve ser incluído para o diagnóstico das ilusões visuais?}

\author{
Seyed-Hossein Abtahi1-3, Mohammad-Ali Abtahit-3
}

1Isfahan Eye Research Center (IERC), Feiz Hospital, Isfahan University of Medical Sciences, Isfahan, Iran;

2Ophthalmology Ward, Feiz Hospital, Isfahan University of Medical Sciences, Isfahan, Iran;

${ }^{3}$ Medical Students Research Center, Isfahan University of Medical Sciences, Isfahan, Iran.

Correspondence: Seyed-Hossein Abtahi; SHARNOS Co. No. 9, Boroomand, Seyed-Alikhan, Chaharbagh Abbasi; 81448-14581 Isfahan - Iran;

E-mail:shf.abtahi@yahoo.com

Conflict of interest: There is no conflict of interest to declare.

Received 24 March 2012; Received in final form 17 April 2012; Accepted 24 April 2012

Dear Editors,

We read with great interest the recent paper by Cerqueira and $\operatorname{Nardi}^{1}$ reporting the association of topiramate (TPM) use and visual illusion in a migraine patient. In our very recent article, we have reviewed the wide range of TPM side effects on vision of 84 patients culled from the literature ${ }^{2}$. As far as we are aware in terms of TPM related visual illusions, five other cases ${ }^{3-5}$ are described in the literature, and the authors of the above report have added the sixth one ${ }^{1}$. It is important to underline that the authors reported a positive dechallenge test that corroborates a causal relationship between TPM and such an adverse effect ${ }^{1,6}$. Nevertheless, in their final conclusion on the etiology of such an association, they stated that "because it may occur in the aura of migraines, these visual illusions are likely to be a result of the migraine"1. Indeed, one of the leading TPM indications is prevention of migraine attacks and herein comes the uncertainty of whether illusions are linked to migraine or to TPM use.

To clarify, firstly, we need to know if TPM associated visual illusions are only observed in migraine consumers. In other words, is there any similar report on a non-migraineur? The authors in their literature review noted two studies reporting illusions in four TPM consumers who were all migraineurs (two cases with palinopsia ${ }^{3}$ and two with Alice-inwonderland syndrome ${ }^{3,4}$ ). Notwithstanding this review, the answer of the above question is positive. Fontenelle, in $2008^{5}$, described palinopsia in a non-migraineur taking TPM for insomnia and impulsive behaviors.

Secondly, we need to know if all of the aforementioned four migraineurs had had history of migraine aura before consumption of TPM. The answer is negative. Only one of them had aura, and in this case no clear temporal relationship was observed between migraine attacks and visual illusions ${ }^{3}$.

Thirdly, it would be rational to adhere to the results of dechallenge and rechallenge tests in this regard. In all of the aforementioned four migraine patients positive dechallenge tests were reported alike what the authors found ${ }^{3,4}$. Moreover, three of these cases were examined by rechallenge tests giving positive results (two palinopsia ${ }^{3}$ and one Alice-in-wonderland syndrome ${ }^{4}$ patients). Engagingly, two of them showed "a clear dose related association with the use of topiramate and the elicitation of palinopsia"3. It is to be noted that rechallenge tests are reputed for being almost the gold standard test in establishing or excluding the etiology of adverse effects ascribed to drugs ${ }^{6}$.

In conclusion, we believe that, along with other drugs, TPM use should be added to the differential diagnosis of visual illusions in migraineurs or non-migraineurs either. Nonetheless, we endorse the notion that the underlying migraine in a vast proportion of TPM consumers can substantially predispose them to these symptoms ${ }^{1,3,4}$.

\section{References}

1. Cerqueira AC, Nardi AE. Metamorphopsia associated with topiramate for migraine prevention. Arq Neuropsiquiatr 2012;70:231-232.

2. Abtahi MA, Abtahi SH, Fazel F, et al. Topiramate and the vision: a systematic review. Clin Ophthalmol 2012;6:117-131.

3. Evans RW. Reversible palinopsia and the Alice in wonderland syndrome associated with topiramate use in migraineurs. Headache 2006;46:815-818.
4.

Jürgens TP, Ihle K, Stork JH, May A. "Alice in Wonderland syndrome" associated with topiramate for migraine prevention. J Neurol Neurosurg Psychiatry 2011;82:228-229.

5. Fontenelle LF. Topiramate-induced palinopsia. J Neuropsychiatry Clin Neurosci 2008;20:249-250.

6. Davidovici BB, Wolf R. The challenge of drug-rechallenge: Facts and controversies. Clin Dermatol 2010;28:349-353. 